

\section{BRASIL E A IDIOSSINCRASIA DA MISCIGENAÇÃO}

EDUARDO TARAZONA-SANTOS ${ }^{1}$ (edutars@gmail.com)

FERNANDA KEHDY ${ }^{1,2}$ (fsgkehdy@gmail.com)

WAGNER CARLOS SANTOS MAGALHÃES ${ }^{1}$ (wCSmagalhaes@gmail.com)

MAÍRA RIBEIRO RODRIGUES' (maira.r.rodrigues@gmail.com)

MARILIA SCLIAR' (mariliascliar@yahoo.com.br)

CAMILA ZOLINI' (camila.Idgh@gmail.com)

MAURICIO L.BARRETO 3 (mauricio@ufba.br)

BERNARDO L. HORTA ${ }^{4}$ (blhorta@gmail.com)

ALEXANDRE C. PEREIRA 5 (alexandre.pereira@incor.usp.br)

MARIA FERNANDA LIMA-COSTA ${ }^{6}$ (lima-costa@cpqrr.fiocruz.br)

RESUmo A iniciativa EPIGEN-Brasil é um projeto estratégico do Ministério da Saúde que constitui a maior iniciativa latino-americana para o estudo da diversidade genômica humana, integrando duas importantes tradições científicas brasileiras: Saúde Coletiva e Genética de Populações. No contexto dos resultados dessa iniciativa, neste artigo discutimos a importância dos estudos de genética de populações para o entendimento do processo de miscigenação no Brasil e para a Saúde Pública. Mostramos como a alta resolução genômica do projeto EPIGEN-Brasil e metodologias computacionais avançadas possibilitaram novas inferências sobre as origens e a dinâmica da miscigenação e como a história social brasileira influenciou a estrutura genética da população.

palavras-chave Genética de populações humanas. História brasileira. Antropologia biológica.

\section{BRAZIL AND THE CROSSBREED IDIOSYNCRASY}

ABSTRACT The EPIGEN-Brazil initiative is a strategic project of the Brazilian Ministry of Health, and is the largest LatinAmerican initiative for the study of human genetic diversity. It integrates two important Brazilian scientific traditions: Public Health and Human Population Genetics. In the context of the results of the EPIGEN-Brazil Project, we discuss how population genetics studies contribute to understand the process of crossbreed in Brazil, and its implications in Public Health. We show how the high genomic resolution of the EPIGEN-Brazil Project and the state-of-the-art computational methodologies allowed new inferences on the origin and dynamics of crossbreed, as well as to understand how the Brazilian social history influenced the genetic structure of this population.

KEYWORDS Human population genetics. Brazilian history. Biological anthropology.

' Departamento de Biologia Geral, Instituto de Ciências Biológicas, Universidade Federal de Minas Gerais.

${ }^{2}$ Laboratório de Hanseníase, Instituto Oswaldo Cruz, Fundação Oswaldo Cruz, FIOCRUZ-RJ.

${ }_{3}^{3}$ Instituto de Saúde Coletiva, Universidade Federal da Bahia.

4 Programa de Pós-Graduação em Epidemiologia, Universidade Federal de Pelotas.

5 Instituto do Coração, Universidade de São Paulo.

${ }^{6}$ Instituto de Pesquisa Rene Rachou, Fundação Oswaldo Cruz.

Recebido em 24/11/2015. Aprovado em 4/4/2016. 
característica mais relevante da população brasileira, como de toda América La-
tina, é a miscigenação, ou, como é mais frequentemente chamada na América hispânica, a mestiçagem (mestizaje). A miscigenação ocorre quando populações que permaneceram isoladas por centenas de gerações passam a se reunir em um espaço geográfico, e indivíduos das diferentes populações de origem se casam e reproduzem. Na América Latina, há pouco mais de 500 anos, desde o encontro entre autóctones nativos americanos, conquistadores europeus (principalmente Ibéricos) e escravos africanos, formou-se uma nova sociedade miscigenada (http://brasil5ooanos.ibge.gov.br/). No Brasil e na América Latina, a grande maioria dos indivíduos é miscigenada em alguma proporção, e existem todas as combinações possíveis de miscigenação individual entre africanos, europeus e nativos americanos. As consequências da miscigenação na biologia, na saúde e em diversos aspectos sociais e culturais têm imensas implicações sobre a nossa história, o nosso cotidiano e o nosso futuro.

A genética de populações parte da análise em laboratório de regiões do DNA que diferem entre indivíduos para estudar a distribuição dessas variantes entre diferentes indivíduos e populações (na terminologia dessa disciplina: determinando a estrutura genética das populações). Com isso, tenta realizar a tarefa mais complexa (porém mais interessante) de inferir os fatores evolutivos atuantes que modelaram a estrutura genética observada. No caso dos estudos de miscigenação, o fator evolutivo mais relevante é o padrão de fluxo gênico (i.e. as migrações). Assim, a genética de populações humanas fornece ferramentas e conceitos para interpretar a miscigenação na América Latina e seus efeitos na saúde, complementando informações de disciplinas, como a história e a demografia histórica, e avaliando o resultado do processo de miscigenação na composição biológica das populações estudadas. Uma característica importante dos estudos de genética de populações atuais é que eles fazem inferências unicamente sobre nossos ancestrais biológicos. Esse enfoque diferencia a genética de populações de outras disciplinas, como a arqueologia ou a história, que podem estudar uma população em um determinado ponto do espaço e do tempo passado, sem ter certeza da medida que os 
indivíduos dessa população são ancestrais da população atual. Por exemplo, podemos estudar dados da origem dos indivíduos europeus desembarcados no Brasil ao longo de um período de tempo, mas essa informação, sendo historicamente relevante, não nos fornece informação sobre a medida que esses indivíduos contribuíram para formar, do ponto de vista biológico, a população brasileira atual. Uma particularidade da história brasileira enfatiza a importância dos estudos de genética de populações. Após a abolição da escravidão em I888, o governo brasileiro ordenou a queima de todos os registros históricos do tráfico de escravos para evitar ações indenizatórias por parte dos antigos donos de escravos (Lacombe et al. I988). Esse fato tem empobrecido o registro histórico sobre migrações de origem Africana no Brasil. No entanto, a genética de populações é uma disciplina que está contribuindo na compensação dessa carência de informações históricas. Assim, a integração das perspectivas de diferentes disciplinas como a história, a demografia, a antropologia física e a genética de populações é necessária para uma interpretação mais completa da história biológica dos brasileiros.

Além da América Latina, existem outras populações miscigenadas, como os afroamericanos e hispanos nos Estados Unidos, os caribenhos (todos esses produto de miscigenação tri-híbrida entre africanos, europeus e nativos americanos), os cabo- verdianos na África (de origem africana e europeia), os coloured na África do Sul (com origens europeia, africana e indiana) e as populações da Ásia Central, exemplificadas em grupos étnicos como os kazakos e uigures, que são produto do encontro entre asiáticos ocidentais e orientais. No entanto, os latino-americanos são modelos clássicos nos estudos da miscigenação em genética de populações humanas e os mais de 200 milhões de brasileiros representam a maior nação miscigenada. Neste artigo, apresentamos resultados do nosso e de outros grupos de pesquisa que tentam responder às seguintes questões: (I) como a história social do Brasil modela a estrutura genética da população brasileira? (2) qual é a origem continental e subcontinental da população brasileira? (3) como aconteceu a dinâmica da miscigenação em diferentes regiões do Brasil?

Determinar a ancestralidade de uma população miscigenada significa inferir as porcentagens dos genomas dos indivíduos miscigenados que são provenientes de cada uma das populações parentais originais. Essas inferências são possíveis devido ao estudo de variantes genéticas presentes na sequência do DNA de cada indivíduo. Para inferir a ancestralidade, a quantidade de informação de cada variante genética 
depende das diferenças das frequências dessas variantes entre as populações parentais que originaram as populações miscigenadas. Variantes genéticas cujas frequências são muito diferentes nas populações parentais são conhecidas como Marcadores Informativos de Ancestralidade (MIAs).

As inferências de ancestralidade em uma população miscigenada podem se dar em três diferentes níveis (Figura I): populacional, individual e cromossômico, sendo que todas essas medidas estão correlacionadas. A ancestralidade populacional é a porcentagem do conjunto dos genomas de uma população (ou como se denominava no passado, o pool génico) que deriva de cada uma das populações parentais. A ancestralidade populacional é a mais fácil de estimar e foi objeto dos primeiros estudos genéticopopulacionais sobre miscigenação. Os primeiros métodos estatísticos para estimar a ancestralidade com base em variantes genéticas usavam a frequência dessas variantes na população miscigenada e nas parentais para estimar a ancestralidade populacional.

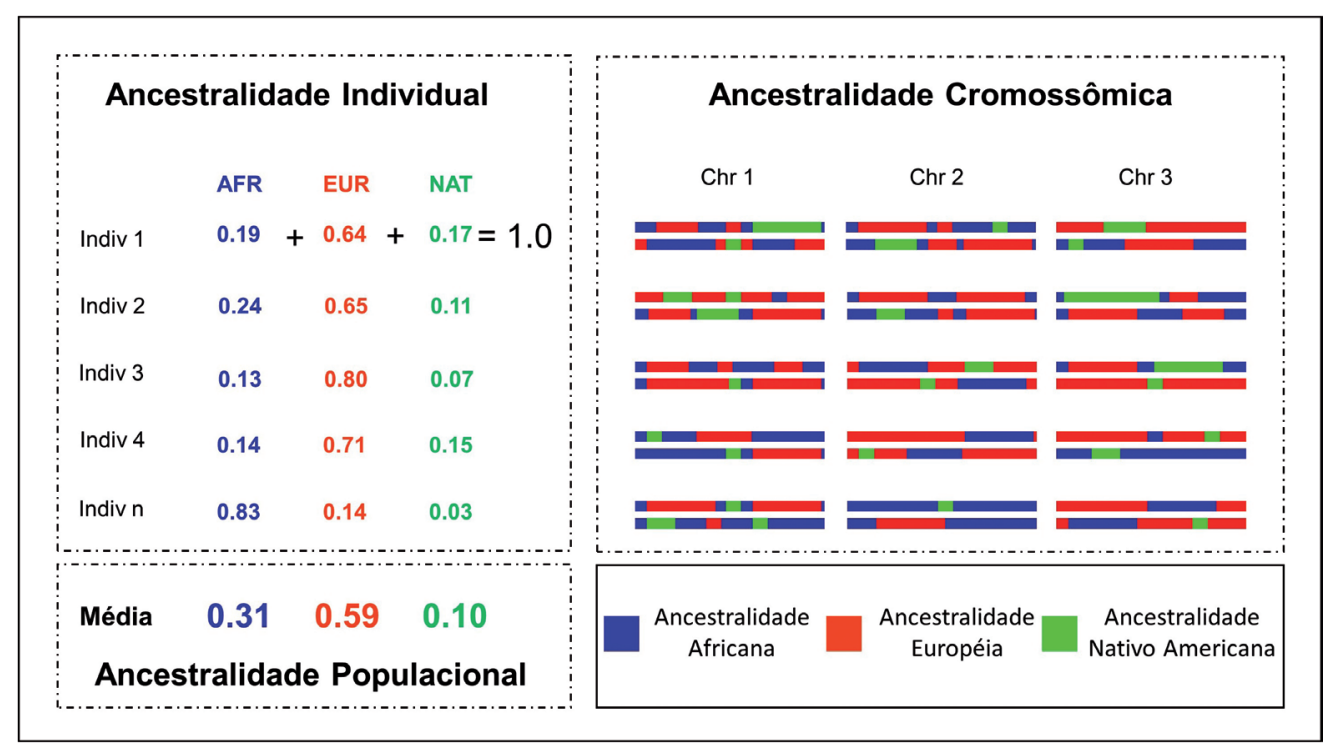

Figura 1. Diferentes níveis de inferência de ancestralidade em uma população miscigenada. Ancestralidade populacional corresponde às porcentagens do conjunto de genomas de todos os indivíduos da população provenientes de cada população parental, ou seja, é a ancestralidade individual média entre todos os indivíduos da população. Ancestralidade individual são as porcentagens do genoma de cada indivíduo originárias de cada população parental. Ancestralidade cromossômica é a origem ancestral de cada fragmento de cada cromossomo de cada indivíduo. 
Com o melhor conhecimento da diversidade do genoma humano e a disponibilidade de mais variantes genéticas para utilização, assim como o desenvolvimento de novos métodos de análise, nos anos 90 passou a ser possível estimar a ancestralidade individual, que consiste nas porcentagens do genoma de um indivíduo miscigenado originárias de cada uma das populações parentais. No contexto da população brasileira, a ancestralidade individual continental (ou tri-híbrida) corresponde às porcentagens africana, europeia e nativo americana do genoma de um indivíduo brasileiro, cujos valores devem somar I. A ancestralidade populacional corresponde à média das ancestralidades individuais entre todos os indivíduos da população.

Em um maior nível de detalhamento, é possível imaginar que os cromossomos dos indivíduos miscigenados são compostos de segmentos de diferentes ancestralidades, constituindo verdadeiros mosaicos de diferentes origens. Com essa ideia em mente, nos últimos anos, devido ao aumento no conhecimento sobre a diversidade do genoma humano em diferentes populações, tornou-se possível utilizar MIAs em alta densidade, ou seja, espalhados por todo o genoma, nas populações parentais e miscigenada, e inferir a ancestralidade de diferentes fragmentos cromossômicos dos indivíduos miscigenados. Essa abordagem é chamada de inferência de ancestralidade cromossômica, ou seja, inferência da ancestralidade de cada ponto de cada cromossomo de cada indivíduo.

O Brasil tem uma importante tradição de estudos em genética de populações humanas publicados em revistas científicas internacionais (Krieger et al. 1965, Freire-Maia I990, Parra et al. 2003, Salzano e Bortolini 2005, Giolo et al. 2012, Kuhn et al. 2012, Moura et al. 20I5). Ainda, na literatura não especializada, existem outras duas sínteses e marcos históricos que tiveram grande influência no contexto cultural brasileiro: o livro Populações Brasileiras, aspectos demográficos, genéticos e antropológicos, publicado em ig 67 pelos Professores Francisco M. Salzano, da Universidade Federal do Rio Grande do Sul, e Newton Freire-Maia, da Universidade Federal do Paraná (Salzano e Freire-Maia I967), e mais recentemente, o artigo Retrato Molecular do Brasil, do grupo dos Professores Sérgio D. Pena e Fabrício R. Santos (Pena et al. 2000), ambos da Universidade Federal de Minas Gerais, publicado quando a miscigenação no Brasil completava 500 anos.

A Saúde Coletiva e a Epidemiologia têm também uma importante tradição de estudos no Brasil. Recentemente, por iniciativa de um grupo de epidemiologistas da 
Fundação Oswaldo Cruz, da Universidade Federal de Minas Gerais, da Universidade Federal da Bahia e da Universidade de Pelotas, com o apoio do Ministério da Saúde e Financiadora de Estudos e Projetos (FINEP), lançou-se a iniciativa estratégica EPIGEN-Brasil (https://epigen.grude.ufmg.br/). Seus objetivos são: (I) estudar em alta resolução genômica a diversidade genética da população brasileira, (2) inferir a origem e dinâmica da miscigenação no Brasil, (3) entender a arquitetura genética de doenças complexas no país e sua relação com a natureza miscigenada da população e (4) entender como variantes genéticas, ambientais e fatores sociais interagem e modelam a susceptibilidade a doenças complexas no país. A iniciativa EPIGEN-Brasil é, antes de tudo, um grande esforço interdisciplinar, que sintetiza grandes tradições científicas brasileiras, de Saúde Coletiva, Epidemiologia e de Genética de Populações, e as integra com outras áreas de pesquisa emergentes no país, como a Bioinformática e a Biologia Computacional. essas áreas são imprescindíveis hoje em dia para analisar a grande quantidade de informação (big data) gerada em projetos que estudam a diversidade do genoma humano em um grande número de indivíduos. Além disso, o projeto EPIGEN-Brasil poderá trazer novas contribuições para compreensão da história da população e das relações raciais no Brasil. A iniciativa EPIGEN-Brasil estuda três entre as mais importantes coortes brasileiras de base populacional, situadas nas três regiões mais povoadas do país: (I) I.246 indivíduos do projeto SCAALA em Salvador/ BA (Barreto et al. 2006), com I8 anos de acompanhamento a partir de I997, quando os participantes eram crianças; (2) I.442 indivíduos da Coorte de Idosos de Bambuí/MG (Lima-Costa et al. 20II), com I8 anos de acompanhamento a partir da linha de base de I997, constituída pelos indivíduos da cidade com mais de 60 anos nessa data; (3) 3.653 indivíduos da Coorte de Nascidos Vivos em ig82 de Pelotas/RS (Víctora e Barros 2006), com 35 anos de acompanhamento. O Projeto EPIGEN-Brasil analisou mais de 2,5 milhões de variantes genéticas em 6.487 participantes das três coortes envolvidas e sequenciou o genoma completo de 30 indivíduos, estabelecendo-se como a maior iniciativa genômica latino-americana. 


\section{O efeito da história social brasileira na estrutura genética da população}

Existem duas características da estrutura genética da população brasileira que refletem a história social do Brasil e da América Latina. Durante os primeiros séculos após a colonização europeia, ocorreu uma alta frequência de casamentos entre homens com ancestralidade predominante europeia e mulheres com ancestralidade predominante africana ou nativa. Isso porque, em parte, nos séculos XVI-XVII relativamente poucas mulheres europeias imigraram para o Novo Mundo. Esse padrão deixou uma assinatura na diversidade genética dos brasileiros que é claramente visível ainda hoje: os cromossomos Y (um marcador de linhagem transmitido patrilinearmente) dos brasileiros são mais frequentemente de origem europeia e o DNA mitocondrial (um marcador de linhagem transmitido matrilinearmente) é mais frequentemente de origem africana ou nativo americana (Carvalho-Silva et al. 2000, Pena et al. 2000, Alves-Silva et al. 2001).

Uma segunda característica da diversidade genômica brasileira e latino-americana é que a ancestralidade continental está correlacionada (mas não necessariamente é a causa) com uma série de características físicas como a cor da pele, com variáveis sociais como a autoclassificação étnico-racial (Ruiz-Linares et al. 20I4, Lima-Costa et al. 20I5), e também com características culturais e socioeconômicas. De fato, na América Latina, os indivíduos com maior ancestralidade africana ou nativa têm menores oportunidades de desenvolvimento e menores níveis socioeconômicos (Telles 2004). Os dados do projeto EPIGEN-Brasil evidenciam que, após cinco séculos de miscigenação, existe ainda uma tendência dos brasileiros de se casarem com indivíduos de ancestralidade similar, o que provavelmente se deve às várias características fenotípicas, culturais e sociais, interligadas com a ancestralidade (Kehdy et al. 20I5). Esse padrão de comportamento matrimonial atua como interface entre cultura e biologia e explica, em parte, por que, após cinco séculos de miscigenação, ainda se observa correlação entre ancestralidade e uma série de fenótipos como a cor da pele e, até mesmo, a classificação étnico-racial. Em termos de modelos de história social e miscigenação, poderíamos pensar em dois modelos extremos de padrões de comportamento matrimonial: um de democracia racial, onde os casamentos se verificam completamente de forma independente da ancestralidade (e as características associadas) e outro de segregação 
racial, que se verificou nos lugares onde esta foi imposta por lei, como o Apartheid na África do Sul ou nos Estados Unidos. Embora no passado o Brasil tenha sido associado a um modelo de democracia racial, por ser sua população mais miscigenada que a afro-americana ou a sul-africana, os estudos sociológicos recentes (Telles 2004) questionam essa interpretação como um "mito", reavaliando o papel das desigualdades raciais e de fenômenos de discriminação na história brasileira e relativizando o papel da miscigenação. Essas interpretações sociológicas mais recentes são coerentes com os resultados do projeto EPIGEN-Brasil supracitados, que ilustram como os casamentos preferenciais, em razão da ancestralidade e características correlacionadas, permearam a história do Brasil. De certa forma, o jornalista Cesar Baima de O Globo (http://oglobo.globo.com/sociedade/ciencia/estudo-diz-que-populacao-do-brasil-menos-misturada-do-que-se-pensa-I660II89), resenhando na versão impressa do jornal a publicação do projeto EPIGEN na revista da Academia Americana de Ciências, sintetizou adequadamente nossos resultados aparentemente paradoxais no contexto de uma sociedade considerada miscigenada por antonomásia, mas não o suficiente para justificar o mito da democracia racial: "Nem tão misturados assim”.

\section{Origem continental e subcontinental da população brasileira}

Os estudos clássicos de genética de populações no Brasil evidenciam, em geral, uma ancestralidade europeia maior no Sul, no Sudeste e no Centro-oeste (nessa ordem); uma ancestralidade africana maior no Nordeste que no Sudeste; e uma ancestralidade indígena baixa no Sul, Sudeste e Nordeste que aumenta no Centro-oeste e no Norte (Pena et al. 20II, Kehdy et al. 2015). Existem afastamentos desse padrão, como as populações de quilombolas, presentes em diversos lugares do país que, como esperado, tem elevada ancestralidade africana (Scliar et al. 2009); como as populações indígenas que, dependendo da localização geográfica e seu histórico de contatos com o Brasil não indígena, apresentam diferentes níveis de ancestralidade não indígena; ou como outros isolados genéticos, cujo estudo sistemático no país está sendo realizado pelo Instituto Nacional de Genética Médica Populacional (Castilla e Schuler-Faccini 
20I4). Os diferentes padrões de ancestralidade observados no Brasil também afetam a distribuição de variantes de importância médica, como aquelas que influenciam a eficácia terapêutica de fármacos e possíveis reações adversas a estes. No Brasil, desde 2003, a Rede Nacional de Farmacogenética (REFARGEN, www.refargen.org,br, SuarezKurtz et al. 20I2) estuda detalhadamente a distribuição dessas variantes no Brasil em razão dos padrões de ancestralidade observados nas diferentes regiões do país.

O projeto EPIGEN-Brasil confirmou esses resultados, exemplificados no Nordeste (Salvador), Sudeste (Bambuí) e Sul (Pelotas) (Figura 2). Adicionalmente, a alta resolução das análises genômicas possibilitou descobrir a ancestralidade dos brasileiros com uma resolução geográfica inédita. Nós evidenciamos que a diversidade genômica de origem europeia dos brasileiros do Sudeste e do Sul tem uma origem geográfica europeia mais ampla e que inclui regiões do Norte da Europa e do Oriente Médio, enquanto que na Região Nordeste a diversidade genômica de origem europeia parece ser restrita à Península Ibérica. Porém, o resultado mais interessante do EPIGEN-Brasil refere-se às origens africanas dos brasileiros, que oferece uma nova perspectiva com base em estudo da população do Brasil, que foi o principal destino da Diáspora Africana. De fato, o Brasil recebeu sete vezes mais escravos africanos (aproximadamente 4 milhões) em comparação aos Estados Unidos (aproximadamente 560 mil), durante um período maior (4 séculos vs. 2 séculos nos Estados Unidos). A origem dos escravos também foi diferente no Brasil em relação ao Caribe e aos Estados Unidos. Enquanto o tráfico de escravos controlado por ingleses, franceses, espanhóis e portugueses teve origem predominante na Costa Ocidental da África, a Coroa Portuguesa era a única estabelecida no Continente africano, particularmente em Moçambique, no Leste da África, de onde também deslocava escravos principalmente para o porto do Rio de Janeiro. Uma questão aberta é se essas origens e destinos diferenciados se refletem ainda na estrutura genética da população brasileira, ou se foram apagados por migrações posteriores de fora do Brasil ou internas. Nós evidenciamos que os brasileiros apresentam pelo menos dois componentes de ancestralidade africana, um deles associado com populações não Bantus do Oeste da África, mais evidente no Nordeste brasileiro, e outro associado com populações Bantu do Leste da África. De maneira interessante, a proporção dessas componentes de ancestralidade que estão presentes em todas as populações africanas e latinas americanas analisadas varia entre elas. O componente 


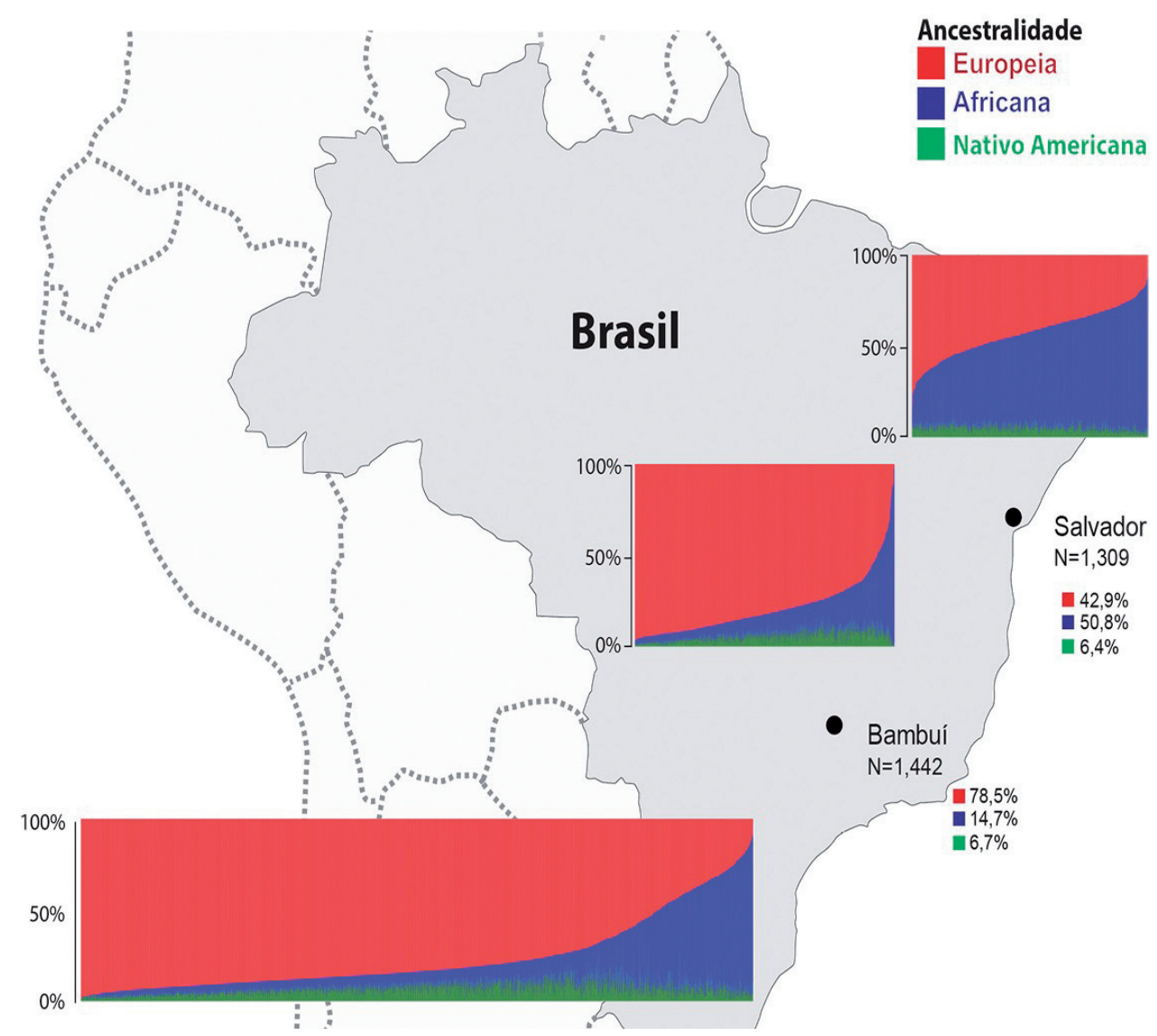

Figura 2. Miscigenação continental das populações estudadas no Projeto EPIGEN-Brasil. Cada retângulo é composto de um conjunto de barras verticais de altura $100 \%$ que representam as ancestralidades individuais em porcentagem: Europeia (vermelha), Africana (azul) e Nativo Americana (verde). N representa o número de indivíduos em cada uma das coortes estudadas. As porcentagens representam a miscigenação continental populacional (média das individuais) para cada uma das coortes. Figura adaptada de Kehdy et al. (2015).

de ancestralidade da África Ocidental/não Bantu é mais evidente nos afro-americanos e no Nordeste do Brasil (Salvador), enquanto o componente da África-Oriental/Bantu é mais evidente no Sudeste e Sul do Brasil, o que verossimilmente é a assinatura ainda presente na diversidade genética dos brasileiros, das migrações de escravos provenientes de Moçambique e regiões próximas ao Rio de Janeiro. Esses resultados evidenciam pela primeira vez uma maior diversidade genética da Diáspora Africana em relação à crença predominante no mundo acadêmico internacional, que considera a África Ocidental como a origem claramente predominante dessa diáspora. Nós observamos que 
essa origem diferenciada determina que pelo menos 38 variantes genéticas que conferem susceptibilidade diferencial a traços e doenças complexas, como doença de Crohn, esclerose múltipla e doença inflamatória do intestino, tem frequências populacionais significativamente diferentes nas duas componentes de ancestralidade africana e entre o Nordeste e Sudeste do Brasil.

\section{Dinâmica da miscigenação no Brasil}

A alta resolução dos dados genômicos produzidos pelo projeto EPIGEN possibilitou ir além das questões clássicas da genética de populações (i.e., quantificar a miscigenação continental) e inferir como ocorreu a miscigenação ao longo do tempo. Para responder a essa questão, usamos informação da ancestralidade cromossômica de cada indivíduo (Figura 4). Ou seja, inferimos ao longo de cada cromossomo de cada indivíduo a origem continental de cada uma das 2,3 milhões de variantes genéticas estudadas. Nós consideramos que os cromossomos de indivíduos miscigenados são mosaicos de fragmentos de ancestralidade europeia, africana e nativo americana, e o fato que, com o passar do tempo, em populações miscigenadas a recombinação genética mistura os cromossomos de diferentes origens, fazendo que a distribuição dos tamanhos dos fragmentos cromossômicos de uma determinada ancestralidade em uma população (chamados tracts na literatura especializada, Figura 3) seja informativa sobre a antiguidade e a história da miscigenação. Utilizando essas informações, implementamos um arcabouço computacional Bayesiano e: (i) inferimos que a pequena miscigenação nativo americana observada no Nordeste, Sudeste e Sul foi introduzida nos primeiros séculos após a colonização europeia, o que é coerente com o fato de que posteriormente as populações nativas foram dizimadas; (ii) evidenciamos a assinatura genômica (tracts de tamanho grande de origem europeu) de imigrações de origem europeia ocorridas nos últimos I50 anos no Sudeste e no Sul, mas não no Nordeste. Isso é coerente com a imigração de europeus nos séculos XIX e XX, em especial nas regiões Sul e Sudeste, favorecida pelo Estado brasileiro, e tendo por fundamento a política de "branqueamento" da população brasileira. 


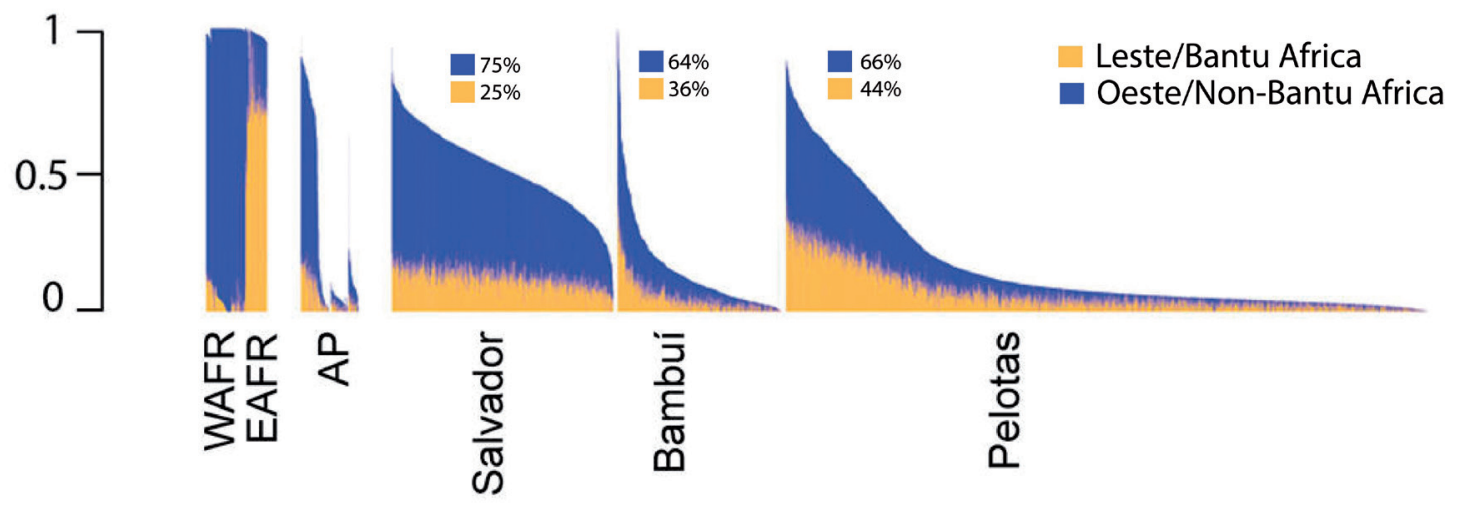

Figura 3. Gráfico de barras verticais representando as ancestralidades subcontinentais Africana nos indivíduos das populações do Projeto EPIGEN-Brasil. Unicamente é representado o componente de ancestralidade Africana para cada indivíduo, subdividido nas porcentagens dos subcomponentes Oeste Africano/não Bantu (azul) e Leste Africano/Bantu). Figura adaptada de Kehdy et al. (2015).

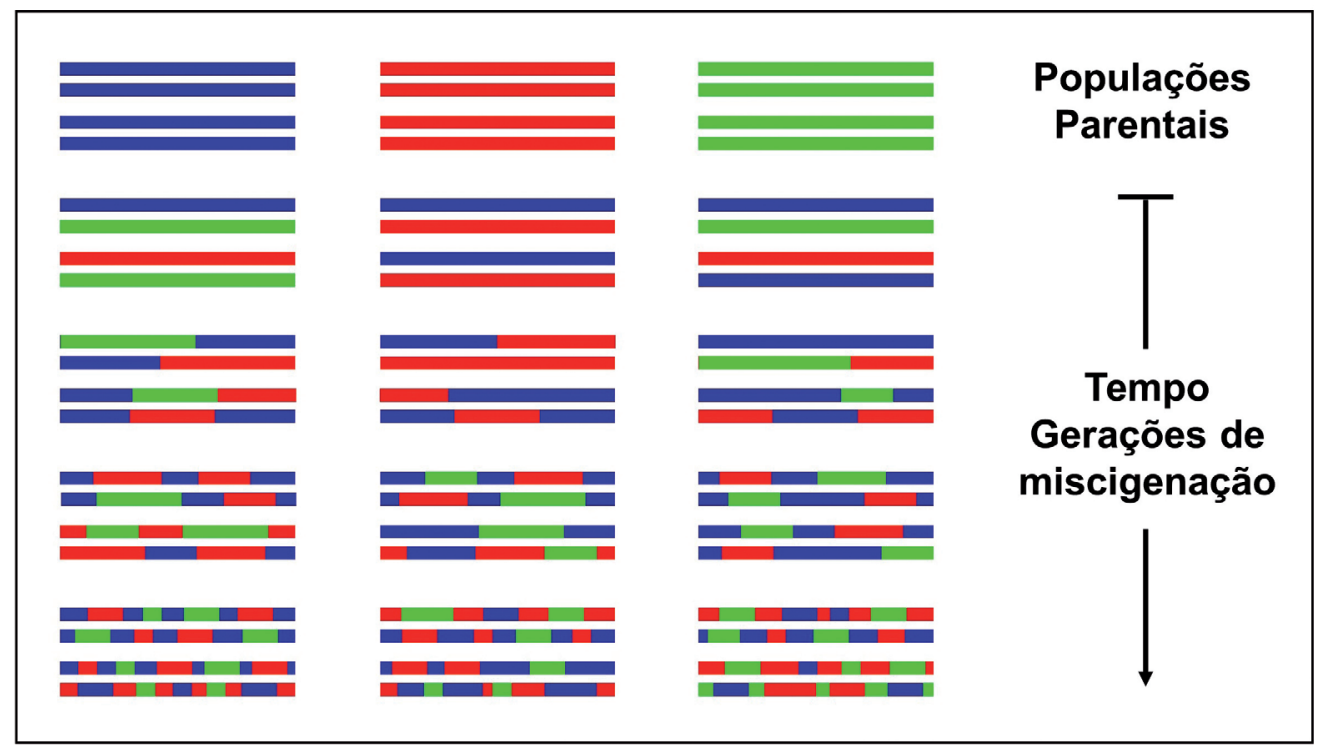

Figura 4. Padrão esquemático da ancestralidade de fragmentos cromossômicos ao longo das gerações de uma população miscigenada gerada de três populações parentais. Cada cor representa uma origem ancestral distinta. Assim que a miscigenação ocorre, os cromossomos dos indivíduos miscigenados são inteiramente (ou quase inteiramente) provenientes de uma única população parental. Devido ao processo de recombinação genética entre cromossomos, os fragmentos cromossômicos de uma determinada ancestralidade vão sendo "quebrados" em pedaços menores à medida que o número de gerações aumenta desde o evento de miscigenação. Dessa forma, indivíduos recentemente miscigenados apresentam fragmentos de determinada ancestralidade mais longos do que os encontrados em indivíduos cuja miscigenação ocorreu há mais gerações. 


\section{Conclusão}

O antropólogo Ricardo Ventura Santos, da Fundação Oswaldo Cruz e do Museu Nacional/UFRJ, tem estudado a trajetória das investigações em genética de populações no Brasil. Ele vem abordando questões sobre a gênese e a trajetória dos estudos de genética de populações brasileiras miscigenadas, desenvolvidos desde os anos I950/60 e como se articulam com questões sobre formação da identidade nacional (de Souza e Santos 20I4). No contexto da América Latina, a genética de populações em uma nação predominantemente miscigenada como o Brasil tem a função social de contribuir com a identidade nacional, assim como a arqueologia pré-hispânica, em países como México e Peru, nas quais a componente de ancestralidade e a herança cultural indígenas são muito mais presentes.

A complexa história de miscigenação no Brasil tem deixado assinaturas que são evidentes na diversidade genômica dos brasileiros, dependendo da resolução genômica em que esta é estudada. Devido à alta resolução genômica dos estudos do projeto EPIGEN-Brasil e tomando-se como referência coortes de base populacional, foi possível revelar novos aspectos em relação ao já grande corpo de conhecimentos gerados pela rica tradição de estudos de genética de populações no Brasil. No ano de ı870, o Brasil era uma nação predominantemente negra, mas esse cenário mudou após a chegada de mais de 4 milhões de europeus na segunda metade do Século XIX e na primeira metade do Século XX. Nós encontramos a assinatura genômica dessas migrações recentes principalmente no Sudeste e Sul do País, onde observamos que a origem geográfica da componente europeia do genoma pode ser reconduzida a uma região mais extensa, que inclui desde Oriente Médio até o Norte de Europa. É interessante que essa grande onda recente de imigrantes europeus, mesmo determinando que a ancestralidade no Sudeste e no Sul em dias atuais seja predominantemente europeia, não tenha apagado dois componentes importantes de ancestralidade africana, presentes e visíveis mesmo entre indivíduos brancos. Um desses componentes de ancestralidade é tradicionalmente associado à ancestralidade Africana Ocidental (predominante no Nordeste). O outro componente de ancestralidade Africana emerge pela primeira vez em estudos de genética de populações relacionados à Diáspora Africana e está associado às migrações de povos do Leste da África, com maior presença no Sudeste e no 
Sul do país. Nossos estudos também evidenciam a tendência ao casamento preferencial em razão da ancestralidade similar, como uma característica que tem permeado a história social brasileira. São necessários ainda estudos adicionais em outras regiões como o Norte e o Centro-oeste, não compreendidos no projeto EPIGEN-Brasil, assim como em outros grandes centros urbanos, além de Salvador (que podem evidenciar uma ancestralidade mais variada, incluindo, por exemplo, a componente japonesa), para ter uma visão mais integrada do processo de miscigenação em todo o Brasil. Ao lado disso, o projeto EPIGEN-Brasil está também focado em estudos orientados a entender como as variantes genéticas presentes no Brasil e produto da miscigenação ocorrida por 5 séculos interagem com o ambiente e modelam a susceptibilidade a traços e doenças complexas como obesidade, hipertensão ou asma.

\section{Agradecimentos}

Os autores agradecem, em particular, ao grupo de pesquisa do Laboratório de Diversidade Genética Humana do Departamento de Biologia Geral da Universidade Federal de Minas Gerais, pelas análises dos dados de miscigenação do projeto EPIGEN. Agradecemos também às equipes dos grupos dos professores Alexandre Pereira, Bernardo Lessa-Horta, Maria Fernanda Lima-Costa e Mauricio Barreto. O DECIT (Departamento de Ciência e Tecnologia) da Secretaria de Ciência, Tecnologia e Estudos Estratégicos do Ministério de Saúde financiaram o Projeto EPIGEN-Brasil. Os autores receberam financiamento da CAPES, CNPq e FAPEMIG. 


\section{Referências}

ALVES-Silva, J.; DA SILVA SANTOS, M.; GUIMARÃES, P.E.; FERREIRA, A.C.; BANDELT, H.J.; PENA,S.D.; Prado, V.F. The ancestry of Brazilian mtDNA lineages. Am J Hum Genet. 67(3):775. Sep 200I.

BARRETO, M.L.; Cunha, S.S.; ALCÂNTARA-NEVES, N.; CARVALHO, L.P.; CRUZ, A.A.; STEIN, R.T.; GENSER, B.; COOPER, P.J.; RODRIGUES, L.C. Risk factors and immunological pathways for asthma and other allergic diseases in children: background and methodology of a longitudinal study in a large urban center in Northeastern Brazil (Salvador-SCAALA study). BMC Pulm Med. 6:I5. Jun 2006.

CARVALHO-SILVA, D.R.; SANTOS, F.R.; ROCHA, J.; PENA, S.D. The phylogeography of Brazilian Y-chromosome lineages. Am J Hum Genet. 68(I):28I-6. Jan 2000.

CASTILLA, E.E.; SCHULER-FACCINI, L. From rumors to genetic isolates. Genet Mol Biol. 2014 Mar; 37 (I Suppl):I86-93.

FREIRE-MAIA, N. Genetic effects in Brazilian populations due to consanguineous marriages. Am J Med Genet. 35(I):II5-7. Jan I990.

GIOLO, S.R.; Soler, J.M.; GREENWAY, S.C.; ALMEIDA, M.A.; de ANDRADE, M.; SEIDMAN, J.G.; SEIDMAN, C.E.; KRIEGER, J.E.; PEREIRA, A.C. Brazilian urban population genetic structure reveals a high degree of admixture. Eur J Hum Genet. 20(I):III-6. Jan 2012.

KEHDY, F.S.; GOUVEIA, M.H.; MACHADO, M.; MAGALHÃES, W.C.; HORIMOTO, A.R.; HORTA, B.L.; MOREIRA, R.G.; LEAL, T.P.; SCLIAR, M.O.; SOARES-SOUZA, G.B.; RODRIGUESSOARES, F.; ARAÚJO, G.S.; ZAMUDIO, R.; SANT ANNA, H.P.; SANTOS, H.C.; DUARTE, N.E.; FIACCONE, R.L.; FIGUEIREDO, C.A.; SILVA, T.M.; COSTA, G.N.; BELEZA, S.; BERG, D.E.; CABRERA, L.; DEBORTOLI, G.; DUARTE, D.; GHIROTTO, S.; GILMAN, R.H.; GONÇALVES, V.F.; MARRERO, A.R.; MUNIZ, Y.C.; WEISSENSTEINER, H.; YEAGER, M.; RODRIGUES, L.C.; BARRETO, M.L.; Lima-Costa, M.F.; PEREIRA, A.C.; RODRIGUES, M.R.; TARAZONA-SANTOS, E.; Brazilian EPIGEN Project Consortium. Origin and dynamics of admixture in Brazilians and its effect on the pattern of deleterious mutations. Proc Natl Acad Sci U S A. I4; II2 (28):8696-70I. Jul 2015.

KOHLRAUSCH, F.; MAGNO, L.A.; MONTENEGRO, R.C.; MORAES, M.O.; de MORAES, M.E.; DE MORAES, M.R.; OJOPI, E.B.; PERINI, J.A.; RACCIOPI, C.; RIBEIRO-DOS-SANTOS, A.K.; RIOS-SANTOS, F.; ROMANO-SILVA, M.A.; SORTICA, V.A.; SUAREZ-KURTZ, G. The genomic ancestry of individuals from different geographical regions of Brazil is more uniform than expected. PLoS One. I6;6 (2):eI7063. Feb 20II.

KRIEGER, H.; MORTON, N.E.; MI, M.P.; AZEVÊDO, E.; FREIRE-MAIA, A.; YASUDA, N. Racial admixture in north-eastern Brazil. Ann Hum Genet. 29(2):II3-25. Nov I965.

KUHN, P.C.; HORIMOTO, A.R.; SANCHES, J.M.; VIEIRA FILHO, J.P.; FRANCO, L.; FABBRO, A.D.; FRANCO, L.J.; PEREIRA, A.C.; MOISES, R.S. Genome-wide analysis in Brazilian Xavante Indians reveals low degree of admixture. PLoS One.7(8):e42702. 2012. 
LACOMBE; A.J.; SILVA, E.; Barbosa, F.A. Rui Barbosa e a Queima dos Arquivos. Rio de Janeiro: Fundação Casa de Rui Barbosa, I988.

LIMA-COSTA, M.F.; FIRMO, J.O.; Uchoa, E. Cohort profile: the Bambui (Brazil) Cohort Study of Ageing. Int J Epidemiol. 40(4):862-7. Aug 20II.

LIMA-COSTA, M.F.; RODRIGUES, L.C.; BARRETO, M.L.; GOUVEIA. M.; HORTA, B.L.; MAMBRINI, J.; KEHDY, F.S.; PEREIRA, A.; RODRIGUES-SOARES, F.; VICTORA, C.G.; TARAZONA-SANTOS, E.; Epigen-Brazil group. Genomic ancestry and ethnoracial selfclassification based on 5,87I community-dwelling Brazilians (The Epigen Initiative). Sci Rep 27;5:9812. Apr 20I5.

MOURA, R.R.; COELHO, A.V.; BALBINO, V.de Q.; CROVELLA, S.; BRANDÃO, L.A. Meta-analysis of Brazilian genetic admixture and comparison with other Latin America countries. Am J Hum Biol. 27(5):674-80. Sep-Oct 20I5.

PARRA, F.C.; AMADO, R.C.; LAMBERTUCCI, J.R.; ROCHA, J.; ANTUNES, C.M., PENA, S.D. Color and genomic ancestry in Brazilians. Proc Natl Acad Sci U S A. 7;I0O(I):I77-82. 30 Dec 2003.

PENA S.D; Carvalho-Silva, D.R.; Alves-Silva, J.; Prado, V.F. Retrato Molecular do Brasil. Ciência Hoje. São Paulo, v. 159, n. 27, Abril, 2000.

PENA, S.D.; Di PIETRO, G.; FUCHSHUBER-MORAES, M.; GENRO, J.P.; HUTZ, M.H.; KEHDY, F.; RUIZ-LINARES, A.; ADHIKARI, K.; ACUÑA-ALONZO, V.; QUINTO-SANCHEZ, M.; JARAMILLO, C.; ARIAS, W.; FUENTES, M.; PIZARRO, M.; EVERARDO, P.; DE AVILA, F.; GÓMEZ-VALDÉS, J.; LEÓN-MIMILA, P.; HUNEMEIER, T.; RAMALLO, V.; SILVA DE CERQUEIRA, C.C.; BURLEY, M.W.; KONCA, E.; DE OLIVEIRA, M.Z.; VERONEZ, M.R.; RUBIO-CODINA, M.; ATTANASIO, O.; GIBBON, S.; RAY, N.; GALLO, C.; POLETTI, G.; ROSIQUE, J.; SCHULER-FACCINI, L.; SALZANO, F.M.; BORTOLINI, M.C.; CANIZALES-QUINTEROS, S.; ROTHHAMMER, F.; BEDOYA, G.; BALDING, D.; GONZALEZ-JOSÉ, R. Admixture in Latin America: geographic structure, phenotypic diversity and self-perception of ancestry based on 7,342 individuals. PLoS Genet. 5;IO(9):eIO०4572. Sep 2014.

SALZANO F.M.; FREIRE-MAIA, N. Populações Brasileiras, aspectos demográficos, genéticos e antropológicos. São Paulo : Cia. Ed. Nacional, Ed. da Universidade I967.

SALZANO F.M.; BORTOLINI, M.C. The Evolution and Genetics of Latin American Populations. Cambridge. Cambridge University Press. 2005.

SCLIAR, M.O.; VAINTRAUB, M.T.; VAINTRAUB, P.M.; FONSECA, C.G. Brief communication: Admixture analysis with forensic microsatellites in Minas Gerais, Brazil: the ongoing evolution of the capital and of an African-derived community. Am J Phys Anthropol.r39(4):59I-5. Aug 2009.

SOUZA, V.S.; SANTOS, R.V. The emergence of human population genetics and narratives about the formation of the Brazilian nation (1950-1960). Stud Hist Philos Biol Biomed Sci. 47:97-107. September 20I4. 
SUAREZ-KURTZ, G.; PENA, S.D.; STRUCHINER, C.J.; HUTZ, M.H. Pharmacogenomic Diversity among Brazilians: Influence of Ancestry, Self-Reported Color, and Geographical Origin. Front Pharmacol. 6;3:191. Nov 2012.

TELLES, E.E. Race in Another America: The Significance of Skin Color in Brazil. New Jersey: Princeton University Press, 2004.

VICTORA, C.G.; BARROS, F.C. Cohort profile: the 1982 Pelotas (Brazil) birth cohort study. Int J Epidemiol. 35(2):237-42. Apr 2006. Epub. Review. Dec 2005. 